\title{
Unilateral pulmonary oedema following the removal of a giant pleural tumour
}

\author{
DAVID A WALLER, NIGEL R SAUNDERS \\ From the Department of Cardiothoracic Surgery, Killingbeck Hospital, Leeds
}

ABSTRACT The occurrence of re-expansion pulmonary oedema immediately after excision of a pleural histiocytoma is described.

Unilateral re-expansion pulmonary oedema is a rare complication of reinflation of a chronically collapsed lung, and may follow treatment of a pneumothorax or drainage of a large pleural effusion.' We report a case of re-expansion pulmonary oedema that followed surgical removal of a giant pleural tumour.

\section{Case report}

A 61 year old woman presented with a two month history of increasing exertional dyspnoea and non-pleuritic right chest pain. Breath sounds were decreased over the right side of the chest, which was uniformly dull to percussion. Chest radiographs showed an opaque right hemithorax with mediastinal shift to the left. Computed tomography of the chest showed a large solid mass occupying the right pleural cavity with underlying lung collapse. Pleural aspiration produced only $10 \mathrm{ml}$ of blood stained fluid. Pleural biopsy showed malignant cells. Right thoracotomy was performed and a large lobulated tumour $(25 \times 20 \mathrm{~cm})$ arising from the visceral pleura of the right upper lobe was removed with pleura and a small margin of underlying lung tissue attached. The right lung was fully inflated manually before closure of the chest. Immediately after reinflation oedema fluid was seen to be exuding from the raw lung surface and endotracheal tube (unfortunately its protein content was not measured). A chest radiograph taken immediately after the operation (fig 1) confirmed the presence of unilateral right sided pulmonary oedema. The patient rapidly developed tachycardia and hypotension. Arterial blood gas analysis indicated substantial hypoxaemia with hypercapnic respiratory acidosis. Treatment was started with a dobutamine infusion, intravenous frusemide, and mechanical ventilation via a single lumen tube with positive end expiratory pressure. There was a good response: a chest radiograph showed considerable improvement in the pulmonary oedema within 12 hours (fig 2) and she was subsequently extubated. Her remaining postoperative course was uncomplicated and she was discharged 13 days after operation. The tumour was found on histological examination to be a malignant fibrous histiocytoma; the underlying lung was compressed but there was no tumour invasion.

Address for reprint requests: Dr David A Waller, Department of Cardiothoracic Surgery, Killingbeck Hospital, Leeds LS14 6UQ.

Fig 1 Chest radiograph 10 minutes after operation, showing unilateral right sided pulmonary oedema.

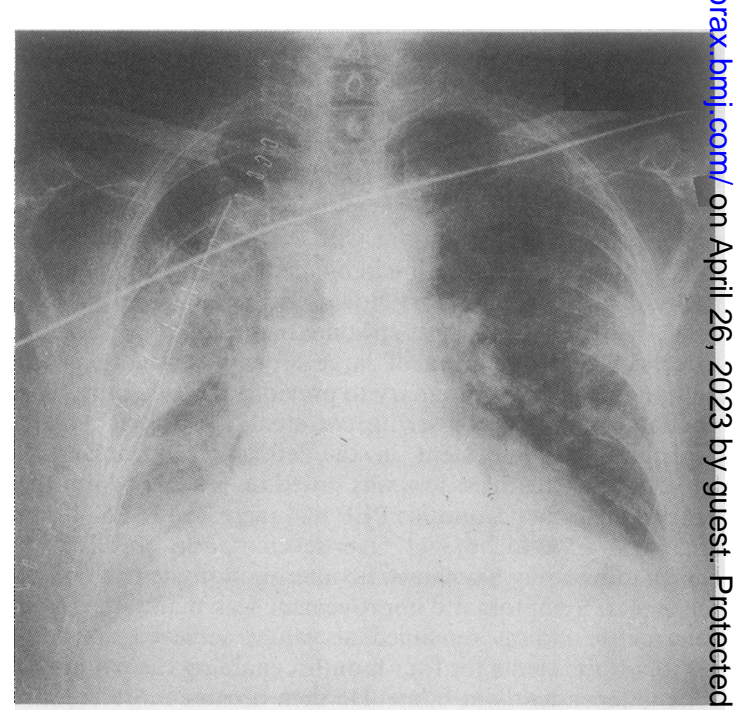

Fig 2 Chest radiograph 12 hours after operation. 


\section{Discussion}

Re-expansion pulmonary oedema is a potentially fatal complication' that may follow the relief of chronic lung collapse. It may follow the rapid evacuation of fluid or air from the pleural cavity. It has also been reported after unilateral bronchial obstruction ${ }^{2}$ and lung compression by a ruptured hemidiaphragm. ${ }^{3}$

The protein content of the oedema fluid is high and increased pulmonary capillary permeability is thought to be the major underlying cause. ${ }^{4}$ Hypoxic damage to the pulmonary microvasculature results from the reduced blood flow in a lung that has usually been collapsed for several days. ${ }^{5}$ This damage is compounded by the rapid increase in blood flow, and consequently in capillary pressure, on re-expansion, and the capillaries may also suffer mechanical stress as they become overdilated. ${ }^{6}$ Pulmonary oedema results from transudation across the leaky alveolar capillary membrane, almost exclusively on the side of previous collapse, although re-expansion oedema has been reported in the contralateral lung; the cause in this case was thought to be capillary damage secondary to systemic hypotension and hypoxaemia compounded by acute pulmonary hypertension.?

Precautions should therefore be taken to retard re-expansion to prevent a sudden drop in pressure across the alveolarcapillary membrane. The use of a large negative intrapleural pressure or forceful reinflation, as in this case, with a large positive airways pressure should be avoided.

Treatment of established re-expansion pulmonary oedema should be directed at correcting hypoxaemia by mechanical ventilation with positive end expiratory pressure and correcting hypotension with plasma expanders and inotropic agents if necessary. Continuous monitoring of arterial and central venous pressures is desirable and arterial blood gas analysis or measurements of oxygen saturation are advisable as radiographic appearances may be misleading. ${ }^{1}$ Hypoxaemia may be severe even though only one lung is affected.

This report shows that re-expansion pulmonary oedema may complicate the removal of a pleural tumour that is causing lung collapse. Awareness of this possibility when such surgery is considered may prevent an unnecessary postoperative complication.

\section{References}

1 Mahfood S. Hix WR, Aaron BL, Blaes P, Watson DC. Re-expansion pulmonary edema. Ann Thorac Surg 1988; 45:340-5.

2 Ravin CE, Dahmash NS. Re-expansion pulmonary edema. Chest 1980;77:708-10.

3 Khoo ST, Chen FG. Acute localised pulmonary oedema. Anaesthesia 1988;43:486-9.

4 Sprung CL, Loewenhesz JW, Baier H, Hauser MJ. Evidence for increased permeability in re-expansion pulmonary oedema. Am J Med 1981;71:497-500.

5 Pavlin DJ. Lung re-expansion-for better or worse? Chest 1986;89:2-3.

6 Pavlin DJ, Nessly ML, Cheney FW. Increased pulmonary vascular permeability as a cause of re-expansion oedema in rabbits. American Review of Respiratory Disease 1981;124: 422-7.

7 Keren A, Tzivoni D, Stern S. Alternating unilateral pulmonary oedema following pneumothorax in acute myocardial infarction. Am Heart J 1983;105:156-9. 